[0212-7199 (2007) 24: 11; pp 551-553] ANALES DE MEDICINA INTERNA Copyright (C) 2007 ARAN EDICIONES, S.L.

AN. MED. INTERNA (Madrid) Vol. 24, N. ${ }^{\circ} 11$, pp. 551-553, 2007

\title{
Síndrome del ácido transretinoico y necrosis cortical renal
}

\author{
A. SASTRE LÓPEZ, E. GAGO GONZÁLEZ, M. BAÑOS GALLARDO, \\ E. GÓMEZ-HUERTAS, F. ORTEGA SUÁREZ \\ Servicio de Nefrología. Hospital Universitario Central de Asturias. Oviedo
}

\begin{abstract}
RESUMEN
Se describe el caso de una paciente con leucemia aguda promielocítica (LAP) que desarrolló síndrome del ácido transretinoico (SAR). El SAR se presenta en enfermos con LAP tratados con ácido transretinoico (ATRA). Tiene incidencia de 5 a 27\% con mortalidad de hasta 29\%. Clínicamente se manifiesta con fiebre, hipotensión, insuficiencia respiratoria, renal y hepática, infiltrados pulmonares, derrame pleural y pericárdico, y edema generalizado. Es secundario al efecto del ATRA sobre la diferenciación de los promielocitos, lo que desencadena respuesta inflamatoria sistémica, daño endotelial con síndrome de fuga capilar y obstrucción de la microcirculación e infiltración tisular. El tratamiento consiste en la suspensión del ATRA, esteroides y medidas de soporte.
\end{abstract}

PALABRAS CLAVE: Ácido trans retinoico. Síndrome del ácido transretinoico. Síndrome del ácido retinoico. Leucemia aguda promielocítica. Respuesta inflamatoria sistémica.
ALL-TRANS RETINOIC ACID SINDROME AND RENAL CORTICAL NECROSIS

\section{ABSTRACT}

We described a patient with acute promyelocytic leucemia (APL) who developed all-trans retinoic acid syndrome (ATRAS). ATRAS presents in patients with APL treated with all-trans retinoic acid (ATRA). It has an incidence from 5-27\% with mortality of 29\%. ATRAS clinical manifestations are fever, hypotension, respiratory, renal and hepatic insufficiency, lung infiltrates, pleural and pericardic efussion, and generalized edema. It is secondary to ATRA effect on promyelocyte differentiation, which causes systemic inflammatory response syndrome, endothelium damage with increase in capillary permeability, microcirculation obstruction, and tissue infiltration. Treatment is based on ATRA suspension, steroids and support measures.

KEY WORDS: All-trans retinoic acid. All-trans retinoic acid syndrome. Retinoic acid syndrome. Acute promyelocytic leukaemia. Systemic inflammatory response syndrome.

Sastre López, A, Gago González E, Baños Gallardo M, Gómez-Huertas E, Ortega Suárez F. Síndrome del ácido transretinoico y necrosis cortical renal. An Med Interna (Madrid) 2007; 24: 551-553.

\section{INTRODUCCIÓN}

La leucemia aguda promielocítica (LAP) corresponde al subtipo M3 de las leucemias mieloides agudas de acuerdo a la clasificación francesa-americano-británica (FAB) y representa $10 \%$ de éstas. Se caracteriza por presentarse en gente joven, ser leucopénica y cursar con elevada incidencia de coagulación intravascular diseminada. Morfológicamente se observan promielocitos hipergranulares con múltiples cuerpos de Auer (1-5). El evento molecular que desencadena la LAP es la fusión del gen del receptor del ácido retinóico que se encuentra en el cromosoma 17q21, a uno de los cuatro genes del cromosoma de la leucemia promielocítica. La fusión mas frecuente y que corresponde $99,9 \%$ de los casos de LAP es la translocación recíproca t $(15 ; 17)(6-8)$. Esto induce leucemo- génesis y hace a la LAP única en sus características genotípicas, inmunofenotípicas, moleculares y de respuesta al tratamiento (9). El ácido transretinoico (ATRA) es un potente inductor de diferenciación y crecimiento celular así como promotor de apoptosis a través de diferentes mecanismos moleculares los cuales activan la expresión y transcripción de genes involucrados en la maduración celular y apoptosis, lo que hace que el uso terapéutico del ATRA a dosis elevadas combinado con quimioterapia sea uno de los mayores avances en el manejo de la LAP, la que es el subtipo de leucemia mieloide aguda con tasa más alta de curación (10-15). El uso de ATRA en los esquemas de manejo de LAP es generalmente bien tolerado, pero algunos enfermos desarrollan el síndrome del ácido transretinoico (SAR) que fue descrito por primera vez en 1992 por Frankel (16).

Trabajo aceptado: 25 de junio de 2007

Correspondencia: Aránzazu Sastre López. Servicio de Nefrología. Hospital Universitario Central de Asturias. C/ Celestino Villamil, s/n. Oviedo, Asturias. e-mail: aranchasastre@hotmail.com 


\section{CASO APORTADO}

Paciente mujer de 29 años de edad sin antecedentes de interés que acude a Urgencias por hematomas cutáneos y hemorragias gingivales. La exploración muestra múltiples hematomas cutáneos. Se realiza hemograma en el que se observan $31 \%$ promielocitos. Ingresa en el Servicio de Hematología donde se diagnostica le LPM, confirmándose la traslocación t $(15,17)$. Se inicia tratamiento según protocolo habitual que incluye ATRA. En el tercer día de tratamiento presentó IRA con anuria. Se realizó ecografía abdominal que mostró riñones de tamaño normal con cortical muy hipercogénica sin dilatación de la vía urinaria. En el Doppler las arterias muestran alta resistencia y escaso flujo cortical. Las venas están permeables. También se objetiva importante ascitis y derrame pleural bilateral. Se suspende el tratamiento con ATRA y se comienza ha realizar hemodiálisis.

Durante esta fase la paciente presenta hemorragia coroidea bilateral que la produce ceguera.

En el día 17 del tratamiento sufrió parada respiratoria que precisó de ingreso en UVI para ventilación mecánica y hemofiltración continua. Siendo alta 14 días después interpretándose el cuadro como de edema agudo de pulmón. En el día 30 del tratamiento reunía criterios de remisión completa, pero continuaba precisando de sesiones de hemodiálisis por diuresis escasa y creatinina elevada, por lo que se decide realizar biopsia renal. En ésta se observa importante edema intersticial, necrosis tubular aguda e imágenes sugestivas de necrosis cortical.

Actualmente ha precisado 2 intervenciones oftalmológicas y ha recuperado parcialmente a visión del ojo derecho.

A los 14 meses del diagnóstico comienza con diálisis peritoneal automatizada y está asintomática. La LPM continúa en remisión completa y todavía recibe tratamiento con ATRA 15 días cada trimestre.

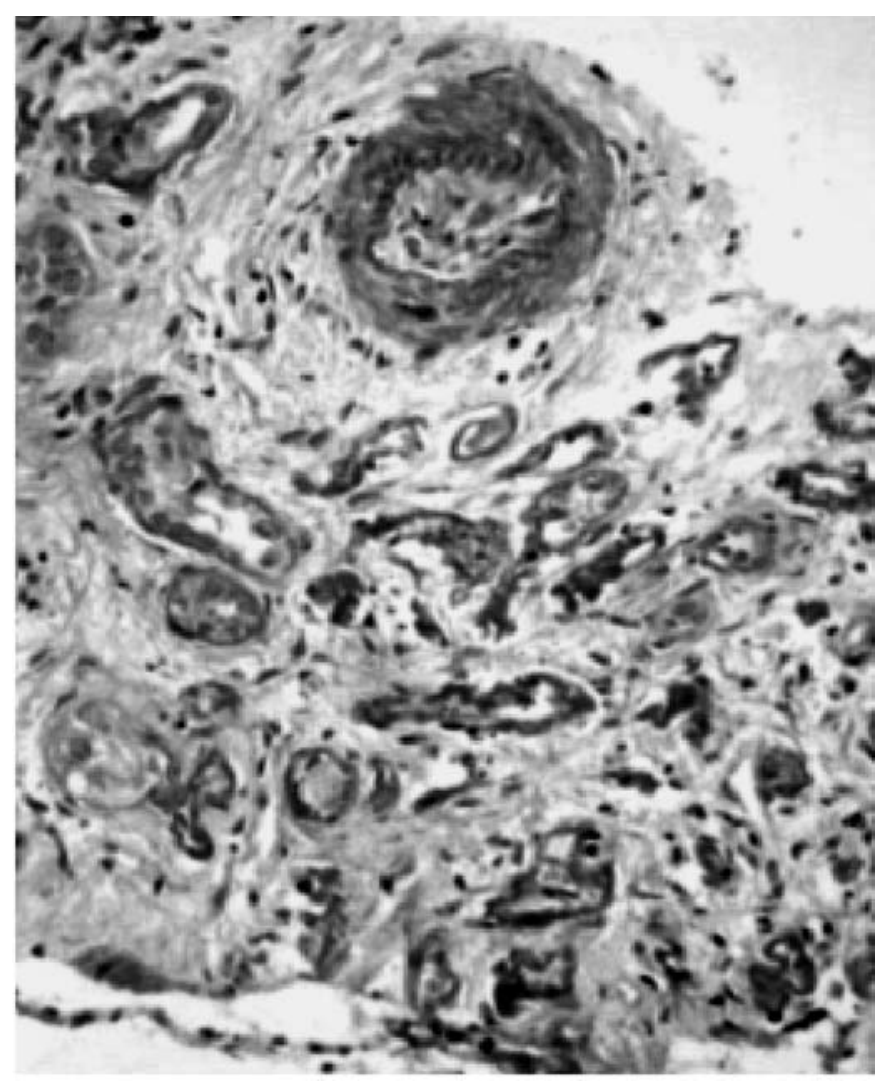

Fig. 1. Necrosis cortical, siluetas glomerulares acelulares, trombo arteriolares, necrosis fibrinoide, túbulos necróticos sin celularidad, membranas basales rotas.

\section{DISCUSIÓN}

El ácido transretinoico tiene efecto proapoptótico e inductor de diferenciación celular y forma parte fundamental del tratamiento de la LAP combinado con otros quimioterápicos. Induce remisiones completas en $92 \%$ de los casos. El SAR es una complicación grave y potencialmente fatal del uso del ATRA en la LAP, su incidencia es de 5 a 27\%, con mortalidad de 5 a $29 \%$. Fue descrito por Frankel y posteriormente corroborado por varios autores (16-25).

Las manifestaciones del SAR son secundarias a tres mecanismos fisiopatogénicos básicos: a) respuesta inflamatoria sistémica; b) daño endotelial con síndrome de fuga capilar y obstrucción de la microcirculación; y c) infiltración tisular. Estos son desencadenados por el efecto del ATRA sobre la diferenciación de promielocitos durante la cual hay síntesis y liberación de interleucinas (IL), proteasas de serina y expresión de moléculas de adhesión endotelial. Los promielocitos expuestos al ATRA forman agregados en la microcirculación con obstrucción de ésta por su interacción con las moléculas de adhesión LFA1-ICAM2 (26-30).El SAR se presenta de 2 a 47 días después de iniciado el tratamiento con ATRA (media de 11 días) y habitualmente se presenta en la fase de diferenciación e incremento de leucocitos y no necesariamente está relacionado a leucocitosis.

El SAR se manifiesta clínicamente con: fiebre, fuga capilar con edema generalizado, insuficiencia respiratoria, infiltrados pulmonares, derrame pleural y pericárdico, vasodilatación, hipotensión, insuficiencia renal aguda e insuficiencia hepática. Histopatológicamente se caracteriza por la infiltración de promielocitos en diversos órganos como hígado, pulmón, riñón, etc., $(31,32)$.El diagnóstico diferencial deberá ser con sepsis debido a que la mayoría de éstos pacientes se encuentran inmunodeprimidos por el efecto de la enfermedad y de la quimioterapia, y a que las manifestaciones clínicas pueden coincidir, para lo cual deberá de descartarse proceso infeccioso y se hará determinación de procalcitonina (32).

El tratamiento para el SAR es a base de suspensión del ATRA, medidas de apoyo, esteroides y leucoféresis. El SAR se manifiesta con respuesta inflamatoria sistémica de diferentes grados de intensidad y disfunción orgánica, por lo que las medidas de apoyo dependerán de los fallos que se presenten.

Insuficiencia renal aguda: en sus fases iniciales es de tipo prerrenal y responde al mejorar el volumen intravascular y la presión de perfusión renal. En su forma grave el SAR cursa con necrosis tubular aguda, en estos casos se requiere de hemodiálisis y en casos más graves y más raros, como el que nosotros presentamos y debido a la fisiopatología del SAR puede cursar con necrosis cortical irreversible.

En conclusión, el SAR es una complicación grave del tratamiento de la LAP con ATRA, es secundario a respuesta inflamatoria sistémica, lesión endotelial e infiltración tisular por promielocitos que se manifiesta con diversas fallos orgánicas, entre ellos el renal. Debe diferenciarse de sepsis grave y del shock séptico. El tratamiento se basa en la suspensión del ATRA, esteroides y medidas de apoyo como la hemodiálisis. 


\section{Bibliografía}

1. Powell BL. Acute progranulocytic leukemia. Curr Opin Oncol 2001; 13: 8-13.

2. Bennett J, Catovsky D, Daniel M, et al. Proposed revised criteria for the classification of acute myeloid leukemia. Ann Intern Med 1985; 103 : 626-9.

3. Stone RM, Mayer RJ. The unique aspects of acute promyelocytic leukemia.J Clin Oncol 1990; 8: 1913-21.

4. Neame PB, Soamboonsrup P, Leber B, et al. Morphology of acute promyelocytic leukemia with cytogenetic or molecular evidence for the diagnosis: Characterization of additional microgranular variants. Am J Hematol 1997; 56: 131-42.

5. Tallman MS, Hakimian D, Kwaan HC, et al. New insights into thepathogenesis of coagulation dysfunction in acute promyelocytic leukemia Leuk Lymphoma 1993; 11: 27-36.

6. Mattson JC. Acute promyelocytic leukemia: From morphology to molecular lesions. Clin Lab Med 2000; 20: 83-103.

7. Melnick A, Licht JD. Deconstructing a disease: RARa, its fusion partners, and their roles in the pathogenesis of acute promyelocytic leukemia. Blood 1999; 93: 3167-215.

8. Mattei MG, Petkovich M, Mattei JF, et al. Mapping of the human retinoic acid receptor to the $\mathrm{q} 21$ band of chromosome 17 . Hum Genet 1988; 80: $186-8$.

9. Wang ZG, Ruggero D, Ronchetti S, et al. PML is essential for multiple apoptoic pathways. Nat Genet 1998; 20: 266-72.

10. Slack JL, Rusiniak ME. Current issues in the management of acute promyelocytic leukemia. Ann Hematol 2000; 79: 227-38.

11. Tallman MS, Andersen JW, Schiffer CA, et al. All-trans-retinoic acid in acute promyelocytic leukemia. N Engl J Med 1997; 337: 1021-8.

12. Fenaux P, Le Deley MC, Castaigne S, et al. European APL 91 Group: Effect of all trans-retinoic acid in newly diagnosed acute promyelocytic leukemia. Results of a multicenter randomized trial. Blood 1993; 82: 3241-9.

13. Fenaux P, Chastang C, Chevret S, et al. European APL Group: A randomized comparison of all transretinoic acid (ATRA) followed by chemotherapy and ATRA plus chemotherapy and the role of maintenance therapy in newly diagnosed acute promyelocytic leukemia. Blood 1999 94: 1192-200.

14. Sanz MA, Martin G, Rayon C, et al. A modified AIDA protocol with antracycline-based consolidation results in high antileukemic efficacy and reduced toxicity in newly diagnosed PML/RARa-positive acute promyelocytic leukemia. Blood 1999; 94: 3015-21.

15. Mandelli F, Diverio D, Avvisati G, et al. Molecular remission in PML/RARapositive acute promyelocytic leukemia by combined alltrans retinoic acid and idarubicin (AIDA) therapy. Blood 1997; 90: 1014-21.

16. Frankel SR, Eardley A, lauwers G, Weiss M, Warrell RP Jr. The "retinoic acid syndrome" in acute promyelocytic leukemia. Ann Intern Med 1992;117:292-296
17. Jonathan KM. The biologic basis for the use of retinoids in cancer prevention and treatment. Curr Opin Oncol 1999; 11: 497-503.

18. Frankel SR, Eardley A, Heller G, Berman E, et al. All-trans retinoic acid for acute promyelocytic leukemia. Ann Intern Med 1994; 120: 278 86

19. De Botton S, Dombret H, Sanz M, San Miguel J, et al. Incidence, clinical features, and outcome of all trans-retinoic acid syndrome in 413 cases of newly diagnosed acute promyelocytic Leukemia. Blood 1998; 8: 2712-8.

20. Fenaux P, Le Deley MC, Castaigne S. Effect of all transretinoic acid in newly diagnosed acute promyelocytic leukemia. Results of a multicenter randomized trial. Blood 1993; 82: 3241-6.

21. Kanamaru A, Takemoto Y, Tanimoto M, Murakami H, et al. All-trans retinoic acid for the treatment of newly diagnosed acute promyelocytic leucemia. Blood 1995; 85: 1202-10

22. Tallman MS, Andersen JW, Schiffer CA, Appelbaum FR. Clinical description of 44 patients with acute promyelocytic leukemia who developed the retinoic acid syndrome. Blood 2000; 95: 90-5.

23. Vahdat L, Maslak P, Miller WH Jr, et al. Early mortality and the retinoic acid syndrome in acute promyelocytic leukemia: Impact of leucocytosis and dose chemotherapy, PML-RARa isoform, and CD13 expression in patients with all-trans retinoic acid. Blood 1994; 84: 3843-9.

24. Asou N, Adachi K, Tamura J, Kanamaru A, et al. Analisis of prognostic factors in newly diagnosed acute promyelocytic leukemia treated with alltrans retinoic acid and chemotherapy. Japan Adult Leukemia Study Group. J Clin Oncol 1998; 16; 78-85.

25. Cortes JE, Kantarjian H, O'Brien S, Robertson LE, et al. All trans retinoicacid followed by chemotherapy for salvage of refractory or relapsed acute promyelocytic leukemia. Cancer 1994; 73: 294-9.

26. Seale J, Delva L, Renesto P, et al. All-trans retinoic acid rapidly decrease cathepsin $\mathrm{G}$ síntesis and mRNA expresión in acute promyelocytic leucemia. Leukemia 1996; 10: 95-101.

27. Di Noto R, Schiavone EM, Ferrara F, Manzo C, et al. All-trans retinoic acid promotes a differential regulation of adhesion molecules on acute myeloid blast cells. Br J Haematol 1996; 88: 247-55.

28. Di Noto R, Schiavone EM, Ferrara F, Manzo C, et al. Expression and ATRA drivenmodulation of adhesion molecules in acute promyelocytic leukemia. Leukemia 1994; 8 (Supl. 2): S71-S76.

29. Dubois C, Schlageter MH, de Gentile A, et al. Modulation of IL-8, IL$1 \mathrm{~b}$, and G-CSF secretion by all-trans retinoic acid in acute promyelocytic leukemia. Leukemia 1994; 8: 1750-7.

30. Marchetti M, Falanga A, Giovanelli S, Oldani E, et al. All-trans retinoic acid increases adhesion to endothelium of the human APML cell line NB4. Br J Haematol 1996; 93: 360-6.

31. Kakkar N. ATRA syndrome with extensive organ infiltration. Am J Hematol 2002; 71: 62-4.

32. Fenaux P, De Botton S. Retinoic acid syndrome. Recognition, prevention and management. Drug Saf 1998; 18: 273-9. 